months in 4 patients and 6 years in one, without apparent development of drug tolerance. (Fink JK et al. Dystonia with marked diurnal variation associated with biopterin deficiency. Neurology May 1988; 38:707-711).

COMMENT: The familial cases described here are similar to those with hereditary progressive dystonia reported by Segawa et al and characterized by diurnal variation and extreme sensitivity to levodopa. (Adv Neurol 1976;14:215). Response to levodopa is generally seen in only $10 \%$ of patients with dystonia.

THERAPY FOR TOURETTE SYNDROME

A 12 year-old male with Tourette Syndrome whose symptoms improved with gum chewing is reported from the Division of Pediatric Neurology, Hahnemann University, Philadelphia, PA. With onset at 3 years of age, symptoms had included blinking, head nodding, neck twisting, shoulder shrugs, snorting, sniffing and blowing, and coprolalia. Haloperidol controlled tics but was associated with excessive drowsiness. Clonidine was of some benefit. After chewing gum, using 2-3 sticks to make a large wad, the urge to move and vocalize was decreased and jaw movements made noise-making more difficult. The author recommends gum chewing as an adjunct therapy in Tourette Syndrome to decrease stress, facial movements, and particularly vocalizations. (Brill CB. Gum chewing as therapy for Tourette syndrome. Pediatr Neurol April 1988; $\underline{4}: 128$ ).

COMMENT: Provided that teachers and parents are tolerant of gum chewing, this form of therapy should be recommended. The prevalence of Tourette syndrome was discussed in a previous issue (Ped Neur Briefs March $1988 ; 2: 24)$. Tics were controlled by haloperidol in 13 of 18 children requiring treatment; the disorder was mild and treatment was unnecessary in 23 (56\%) patients (Neurology $1988 ; 88: 472$ ).

\title{
LESCH-NYHAN SYNDROME
}

Five boys with Lesch-Nyhan syndrome and varying degrees of dystonia, chorea, spasticity, ataxia, dysarthria, and mental retardation were studied at the Depts of Neurology and Medicine, Baylor College of Medicine, and Depts of Neurology and Pediatrics, University of Texas Health Science Center, Houston, TX. Four showed reduction of homovanillic acid (HVA) in the CSF and all had low CSF phenethylene glycol, indicating reducee dopamine and norepinephrine turnover. Three children had high CSF 5-hydroxyindoleacetic acid (HIAA), suggesting increased serotonin turnover. The patient with the most severe chorea had the lowest CSF HVA value, whereas the patient with the least amount of self-mutilation had the highest CSF 5HIAA. Only one patient improved with carbidopa-levodopa, whereas all 5 showed some lessening of self-mutilatory or hyperkinetic behavior in response to tetrabenazine, a monoamine-depleting agent. The study was thought to support the theory of abnormal central monoamine metabolism in Lesch-Nyhan syndrome. (Jankovic J et al. Lesch-Nyhan syndrome: A study of motor behavior and cerebrospinal fluid neurotransmitters. Ann Neurol May 1988; 23:466-469).

COMMENT: Lesch-Nyhan syndrome is an $\mathrm{x}$-linked recessive disorder 
of purine metabolism characterized by hypotonia followed by spasticity, chorea, athetosis, dystonia, growth and mental retardation, self-mutilatory behavior, hyperuricemia, and nephrolithiasis (Lesch M, Nyhan WL. AM J Med 1964;36:561). The phenotypical expression is the result of a deficiency of hypoxanthineguanine phosphoribosyl-transferase enzyme, the gene located on the long arm of the $\mathrm{x}$ chromosome. Response to medications is variable but self-mutilation can sometimes be ameliorated (Herman $\mathrm{BH}$ et al. Naltrexone decreases self-injurious behavior. Ann Neurol $1987 ; 22: 550$ ).

\section{INIRACRANIAL TUMORS}

NEONATAL CRANIOPHARYNGIOMA

A craniopharyngioma detected in utero using ultrasound is reported from the Depts of Neurosurgery, Neonatology, Pathology, and Obstetrics and Gynecology, New York University Medical Center, New York, NY. The obstetric ultrasound study performed because of premature labor at 35 weeks showed polyhydramios and macrocephaly secondary to an intracranial, calcified lobulated mass. A CT scan performed at $2 \mathrm{hrs}$. of life demonstrated a calcified intracranial mass extending to the base of the skull. The infant suffered cardiorespiratory arrest and died at the second day of life. At postmortem examination, the suprasellar mass was a granular tumor with multiple cysts filled with green mucoid material. Microscopically, the tumor showed branching cords and pallisading of epithelial cells, focal calcifications, and an inner zone of stellate cells, an appearance diagnostic of craniopharyngioma. The authors uncovered only four other reports of congenital intracranial neoplasms diagnosed prenatally using ultrasound. Three were teratomas and one a dysplastic mass. Calcifications have been observed in teratomas and meningiomas in utero and are not pathognomonic of craniopharyngioma. Low set ears and polydactyly are reported as associated congenital anomalies. Polyhydramnios occurred in all five cases of neonatal brain tumors diagnosed antenatally by ultrasound. Radical excision of the tumor in the neonatal period was not advised. (Freeman TB et al. Neonatal cranipharyngioma. NY State J Med Feb 1988;88:81-83).

COMMENT: Craniopharhngiomas comprise about $3 \%$ of all intracranial tumors at all ages and $9 \%$ in children. Only 10 cases of neonatal craniopharyngioma were culled from the literature. Radical excision for cases presenting in infancy is recommended by the following authors reporting their experiences with 50 cases in Paris.

\section{TREATMENT OF CRANIOPHARYNGIOMA IN INFANCY}

A retrospective analysis of the outcome of 50 cases of craniopharyngioma treated in infancy by radical or subtotal surgical excision and irradiation is reported from the Services of Neurosurgery and Endocrinology, Hopital des Enfants Malades, 149, reu de Sevres, 75743 Paris Cedex 15, France. The authors concluded that 1) radical excision is the treatment of choice; 2) if radical excision is not possible, surgery should be followed by irradiation to lower risk of recurrence; and 3 ) radiotherapy should be delayed as long as possible because of hazards to 